\title{
Tight Closed-Form Approximation for the Ergodic Capacity of Orthogonal STBC
}

\author{
Jesús Pérez, Member, IEEE, Jesús Ibáñez, Member, IEEE, Luis Vielva, David J. Pérez-Blanco, \\ and Ignacio Santamaría, Senior Member, IEEE
}

\begin{abstract}
In this letter we derive a simple and tight closedform approximation for the ergodic capacity of orthogonal spacetime block coding in arbitrary fading channels. The expression is an analytical function of the power covariance matrix of the channel. In the case of uncorrelated channels the expression only depends on the variances of the channel power gains. These channel statistics can be easily obtained from both analytical and physical fading channel models. Simulations results show the accuracy of the proposed expression.
\end{abstract}

Index Terms-MIMO systems, channel capacity, space-time block codes (STBCs), fading channels.

\section{INTRODUCTION}

$\mathbf{O}$ RTHOGONAL Space-Time Block Coding (OSTBC) is able to provide full diversity gain over i.i.d. complex Gaussian multiple-input-multiple-output (MIMO) channels, with very simple encoding and decoding [1], [2]. In contrast to other space-time codes, OSTBC schemes do not provide coding gain, unless concatenated with an outer code. When OSTBC is concatenated with standard AWGN codes, the overall system performance is similar to, or even better than more complex space-time coding schemes like space-time trellis codes [3]. Usually, the bit error rate is employed as a performance metric. But, when the OSTBC is concatenated with outer codes, the system capacity is an adequate metric for the overall system design. The performance of MIMO-OSTBC systems strongly depends on the specific MIMO channel characteristics. Closed-form expressions for both outage and ergodic capacity have been derived for specific analytical fading channel models like Rayleigh, Ricean [4], [5] and Nakagami channels [6].

In this paper we derive a simple and tight approximate closed-form expression for the ergodic capacity of MIMOOSTBC systems that is valid for arbitrary channel fading distributions. This expression is a function of the variance of the squared Frobenius norm of the channel matrix, which can be expressed as a simple function of the power covariance matrix. In the case of uncorrelated channels the expression only depends on the variances of the channel power gains. These channel statistics can be easily obtained from both analytical (non-physical) and physical fading channel models. Therefore the proposed expression can be used in conjunction with both

Manuscript received May 22, 2005; revised August 9, 2005; accepted December 2, 2005. The associate editor coordinating the review of this paper and approving it for publication was H. Jafarkhani.

The authors are with the Universidad de Cantabria, ETSIIyT, Avda. de los Castros S/N, Santander, Cantabria 39005, Spain (e-mail: \{jperez, jesus, blanco,nacho\}@gtas.dicom.unican.es; luis@dicom.unican.es).

Digital Object Identifier 10.1109/TWC.2007.05337. types of channel modeling. Simulation results demonstrate the accuracy of the approximation regardless of the fading distribution, correlation conditions, signal-to-noise ratio and number of antennas.

\section{Signal Model}

We assume a quasi-static and frequency-flat fading channel, which is known at the receiver but unknown at the transmitter. We also assume i.i.d. AWGN noise at the receive antennas. Considering the base-band equivalent signal model, the received signal vector is given by

$$
\mathbf{y}=\sqrt{\alpha} \mathbf{H} \mathbf{x}+\mathbf{n}
$$

where $\mathbf{y}$ is a $n_{R} \times 1$ vector representing the signals at the receive antennas, $\mathbf{x}$ is the $n_{T} \times 1$ vector transmitted by the $n_{T}$ transmit antennas, $\alpha$ is the average path-loss between the transmitter and the receiver, and $\mathbf{H}$ is the normalized $n_{R} \times n_{T}$ MIMO channel matrix, so the expected value of the squared Frobenius norm of the channel matrix is $E\left[\|\mathbf{H}\|_{F}^{2}\right]=n_{R} n_{T}$. $\mathbf{n}$ is the $n_{R} \times 1$ noise vector with zero-mean circularly symmetric i.i.d. complex Gaussian random entries with variance $\sigma^{2}$.

After OSTBC decoding [1], [2], [7] the system is equivalent to $k$ independent scalar AWGN subchannels given by

$$
z_{i}=\frac{1}{R} \alpha\|\mathbf{H}\|_{F}^{2} s_{i}+w_{i}, i=1, . ., k
$$

where $k$ is the number of symbols of the OSTBC block, $z_{i}$ is the scalar processed received signal corresponding to symbol $s_{i}$ and $w_{i}$ is a zero-mean circularly symmetric complex Gaussian random variable with variance $\alpha\|\mathbf{H}\|_{F}^{2} \sigma^{2} / R$, where $R$ is the code rate. The effective signal-to-noise ratio at detection is given by [7], [8], [9], [10]

$$
s n r=\frac{E_{s} \alpha\|\mathbf{H}\|_{F}^{2}}{\sigma^{2} n_{T} R}=\rho \frac{\|\mathbf{H}\|_{F}^{2}}{n_{T} R}
$$

where $E_{s}$ is the total transmitted energy on the $n_{T}$ transmit antennas per symbol time and $\rho=E_{s} \alpha / \sigma^{2}$ is the average signal-to-noise ratio at the receiver branches before decoding.

\section{ERGODIC CAPACITY}

The capacity (in bps/Hz) can be expressed as [7]- [10]

$$
C=R \log _{2}(D), \quad D=1+s n r .
$$


To derive a closed-form approximation for the ergodic capacity, we first expand (4) in a Taylor series about the expected value of $D$ in (4)

$$
\begin{aligned}
C(D) & =R \log _{2} E[D]-R \log _{2}(e) \\
& \times \sum_{m=1}^{M} \frac{(-1)^{m}}{m} \frac{(D-E[D])^{m}}{E[D]^{m}}+R_{M+1}(D)
\end{aligned}
$$

where $e$ is the Neper's number and $R_{M+1}$ is the series remainder which can be expressed in the Lagrange form as follows [11]

$$
R_{M+1}(D)=R \log _{2}(e) \frac{(-1)^{M}}{M+1} \frac{(D-E[D])^{M+1}}{\beta^{M+1}}
$$

where $\beta$ is between $D$ and $E[D]$. When $M$ is odd, $R_{M+1}$ is negative, so the truncated $M^{\text {th }}$-order approximation is an upper-bound of the capacity for any value of $D$. Applying the expectation operator to (5), the $M^{t h}$-order approximation for the ergodic capacity will be

$$
\begin{aligned}
E[C] & \approx R \log _{2} E[D] \\
& -R \log _{2}(e) \sum_{m=2}^{M} \frac{(-1)^{m}}{m} \frac{E\left[(D-E[D])^{m}\right]}{E[D]^{m}} .
\end{aligned}
$$

Note that the $m=1$ term in (5) is zero after the expectation is taken. Considering (3), (4) and the channel normalization, the expected value and the central moments of $D$ can be expressed as follows

$$
\begin{gathered}
E[D]=1+\frac{\rho}{n_{T} R} E\left[\|\mathbf{H}\|_{F}^{2}\right]=1+\frac{\rho n_{R}}{R}, \\
E\left[(D-E[D])^{m}\right]=\left(\frac{\rho}{n_{T} R}\right)^{m} \\
\times E\left[\left(\|\mathbf{H}\|_{F}^{2}-E\left[\|\mathbf{H}\|_{F}^{2}\right]\right)^{m}\right] .
\end{gathered}
$$

Then, (7) is an approximation of the ergodic capacity as a function of the moments of $\|\mathbf{H}\|_{F}^{2}$. Considering that $C$ is a concave function of $D$ (see (4)), the Jensen's inequality provides the following upper-bound for the ergodic capacity $E[C] \leq R \log _{2} E[D]$. This upper-bound coincides with the first term in (7). The second-order approximation $(M=2$ in (7)) reduces to

$$
E[C] \approx R \log _{2}\left(1+\frac{\rho n_{R}}{R}\right)-\frac{R \log _{2}(e) \rho^{2} \operatorname{var}\|\mathbf{H}\|_{F}^{2}}{2 n_{T}^{2}\left(R+\rho n_{R}\right)^{2}}
$$

where var $\|\mathbf{H}\|_{F}^{2}$ is the variance of the squared Frobenius norm of $\mathbf{H}$.

\section{Analytical fading CHANNEL MODELS}

In the second-order approximation of (10), the ergodic capacity only depends on the variance of the squared Frobenius norm of $\mathbf{H}$. It can be expressed as follows

$$
\begin{aligned}
\operatorname{var}\|\mathbf{H}\|_{F}^{2} & =\sum_{i=1}^{n_{R}} \sum_{j=1}^{n_{T}} \sum_{k=1}^{n_{R}} \sum_{s=1}^{n_{T}} E\left[\left|h^{i j}\right|^{2}\left|h^{k s}\right|^{2}\right] \\
& -E\left[\left|h^{i j}\right|^{2}\right] E\left[\left|h^{k s}\right|^{2}\right]
\end{aligned}
$$

where the $h^{i j}$ denote the entries of $\mathbf{H}$. The variance of $\|\mathbf{H}\|_{F}^{2}$ equals the sum of the entries of the power covariance matrix $\mathbf{R}_{P}$, that are given by [12]

$$
\rho_{P}^{i j, k s}=E\left[\left|h^{i j}\right|^{2}\left|h^{k s}\right|^{2}\right]-E\left[\left|h^{i j}\right|^{2}\right] E\left[\left|h^{k s}\right|^{2}\right] .
$$

Therefore, the power covariance matrix is the only channel statistic required for the capacity estimation. Much of the experimental research in antenna diversity has involved the measurements of power covariance matrices.

In the case of uncorrelated channels $\mathbf{R}_{P}$ is diagonal, so the variance reduces to

$$
\operatorname{var}\|\mathbf{H}\|_{F}^{2}=\operatorname{Tr}\left(\mathbf{R}_{P}\right)=\sum_{i=1}^{n_{R}} \sum_{j=1}^{n_{T}} \rho_{P}^{i j, i j}=\sum_{i=1}^{n_{R}} \sum_{j=1}^{n_{T}} \operatorname{var}\left|h^{i j}\right|^{2}
$$

where $\operatorname{Tr}(\cdot)$ denotes the trace of the corresponding matrix. Therefore, in this case we only need the power variances of the fading distributions. These variances can be obtained analytically for most fading distributions. Assuming that the $\Omega_{i j}$ are the mean powers of the channel matrix entries (they determine the potential imbalances of the channel), the variances for Nakagami-m, Rice and Weibull fading distributions are given by

$$
\begin{gathered}
\operatorname{var}\left|h^{i j}\right|^{2}=\frac{\Omega_{i j}^{2}}{m_{i j}} \\
\operatorname{var}\left|h^{i j}\right|^{2}=\Omega_{i j}^{2}\left(\frac{2 \exp \left(-K_{i j}\right)_{1} F_{1}\left(3 ; 1 ; K_{i j}\right)}{\left(K_{i j}+1\right)^{2}}-1\right) \\
\operatorname{var}\left|h^{i j}\right|^{2}=\Omega_{i j}^{2}\left(\frac{\Gamma\left(1+4 / \beta_{i j}\right)}{\left(\Gamma\left(1+2 / \beta_{i j}\right)\right)^{2}}-1\right)
\end{gathered}
$$

where the $m_{i j}$ are the Nakagami parameters, the $K_{i j}$ are the ricean factors and the $\beta_{i j}$ are the Weibull parameters. ${ }_{1} F_{1}(\cdot ; \cdot ; \cdot)$ is the confluent hypergeometric function and $\Gamma(\cdot)$ is the gamma function. The Rayleigh distribution can be viewed as a particular case of the Nakagami distribution with $m_{i j}=1$, Ricean distribution with $K_{i j}=0$ or Weibull distribution with $\beta_{i j}=2$. Similarly, other fading distributions can be considered. Combining (14), (15) or (16) with (13) and (10), we can estimate the ergodic capacity in these fading channels.

The approximation in (10) can be employed when the channel matrix entries have different gains and fading distributions. This situation can arise in distributed communication networks employing cooperative STBC transmission [6], [13]. In these cases the variance of each power gain in (13) will be calculated according to the corresponding mean power gain $\Omega_{i j}$, fading distribution and fading parameter.

\section{Physical fading Channel Model}

The expression (10) can also be used in conjunction with physical channel models. Here we use the narrow-band ISLAC (Independent-Stochastic Local Area Channel) model [14]. This is a physical fading channel model that encompasses a wide variety of fading distributions, including the Rayleigh and Ricean cases. According to this model, the base-band equivalent MIMO channel matrix $(\mathbf{H})$ can be expressed as the sum of the contributions of $N$ multi-path matrices $\left(\mathbf{V}_{n}\right)$ 
corresponding to $N$ uniform plane waves due to the multi-path propagation,

$$
\mathbf{H}=\sum_{n=1}^{N} \exp \left(j \phi_{n}\right) \mathbf{V}_{n}
$$

where the $\phi_{n}$ are independent uniformly distributed random phases, each one associated with a wave. The $\mathbf{V}_{n}$ are deterministic matrices that depend on the array configuration, antenna characteristics, and the transmit and receive angular power spectra in the specific propagation scenario. They can be obtained from measurements, from radio-propagation simulation tools or from given angle power spectra [14].

The specific values of the multi-path matrices $\mathbf{V}_{n}$ completely determine the statistical characteristics of $\mathbf{H}$ including fading distributions of the channel matrix entries, correlation, gain imbalances, etc. Considering the statistical distribution of the phases $\phi_{n}$, the covariance matrix of the channel can be expressed analytically as a function of the multi-path matrices as follows

$$
\mathbf{R}=E\left[\operatorname{vec}(\mathbf{H}) \operatorname{vec}(\mathbf{H})^{H}\right]=\sum_{n=1}^{N} \operatorname{vec}\left(\mathbf{V}_{n}\right) \operatorname{vec}\left(\mathbf{V}_{n}\right)^{H}
$$

where vec (.) is the vectorization operator and the superscript $(.)^{H}$ denotes conjugate transpose.

From (17), the squared Frobenius norm of the channel matrix can be expressed as follows

$$
\begin{aligned}
\|\mathbf{H}\|_{F}^{2} & =\operatorname{Tr}\left(\mathbf{H} \mathbf{H}^{H}\right) \\
& =\sum_{n=1}^{N} \sum_{m=1}^{N} \operatorname{Tr}\left(\mathbf{V}_{n} \mathbf{V}_{m}^{H}\right) \exp \left(j\left(\phi_{n}-\phi_{m}\right)\right)
\end{aligned}
$$

Considering the statistical distribution of the phases, $\phi_{n}$, the moments of $\|\mathbf{H}\|_{F}^{2}$ can be expressed analytically as a function of the multi-path matrices. For example, the mean of $\|\mathbf{H}\|_{F}^{2}$ will be given by

$$
\begin{aligned}
E\left[\|\mathbf{H}\|_{F}^{2}\right] & =\sum_{n=1}^{N} \sum_{m=1}^{N} \operatorname{Tr}\left(\mathbf{V}_{n} \mathbf{V}_{m}^{H}\right) E\left[\exp \left(j\left(\phi_{n}-\phi_{m}\right)\right]\right. \\
& =\sum_{n=1}^{N} \operatorname{Tr}\left(\mathbf{V}_{n} \mathbf{V}_{n}^{H}\right)=\sum_{n=1}^{N}\left\|\mathbf{V}_{n}\right\|_{F}^{2}
\end{aligned}
$$

Similarly, considering

$$
\begin{aligned}
\|\mathbf{H}\|_{F}^{4} & =\sum_{n 1=1}^{N} \sum_{m 1=1}^{N} \sum_{n 2=1}^{N} \sum_{m 2=1}^{N} \operatorname{Tr}\left(\mathbf{V}_{n 1} \mathbf{V}_{m 1}^{H}\right) \operatorname{Tr}\left(\mathbf{V}_{n 2} \mathbf{V}_{m 2}^{H}\right) \\
& \times \exp \left(j\left(\phi_{n 1}+\phi_{n 2}-\phi_{m 1}-\phi_{m 2}\right)\right.
\end{aligned}
$$

the second moment of $\|\mathbf{H}\|_{F}^{2}$ can be expressed as follows

$$
\begin{aligned}
E\left[\|\mathbf{H}\|_{F}^{4}\right] & =\sum_{n=1}^{N} \sum_{m=1}^{N}\left\|\mathbf{V}_{n}\right\|_{F}^{2}\left\|\mathbf{V}_{m}\right\|_{F}^{2} \\
& +2 \sum_{n=1}^{N} \sum_{m>n}^{N}\left|\operatorname{Tr}\left(\mathbf{V}_{n} \mathbf{V}_{m}^{H}\right)\right|^{2}
\end{aligned}
$$

Then, the variance of $\|\mathbf{H}\|_{F}^{2}$ will be given by

$$
\begin{aligned}
\operatorname{var}\|\mathbf{H}\|_{F}^{2} & =E\left[\|\mathbf{H}\|_{F}^{4}\right]-E\left[\|\mathbf{H}\|_{F}^{2}\right]^{2} \\
& =2 \sum_{n=1}^{N} \sum_{m>n}^{N}\left|\operatorname{Tr}\left(\mathbf{V}_{n} \mathbf{V}_{m}^{H}\right)\right|^{2} .
\end{aligned}
$$

Notice that the first and second moments of $\|\mathbf{H}\|_{F}^{2}$ are simple functions of the multi-path matrices $\mathbf{V}_{n}$. Then, the approach in (10) can be employed to yield a closed-form approximation for the ergodic capacity as a function of the multi-path matrices.

The channel model of (17) can also be viewed as a tool to model specific MIMO fading channels by the adequate selection of the multi-path matrices. This allow us to model a wide variety of fading distributions (that can be different for different entries of $\mathbf{H}$ ), gain imbalances and correlations. Here, as examples, we will focus on the modeling of Rayleigh and Ricean fading distributions.

\section{A. Physical model of Rayleigh fading channels}

Let $V_{n}^{i j}$ denote the entry of $\mathbf{V}_{n}$ corresponding to the $i$-th receive and the $j$-th transmit antenna. When $N$ is large and the amplitudes of the $V_{n}^{i j}$ (for fixed $i$ and $j$ ) are similar, the quantity $\left|h^{i j}\right|$ exhibits Rayleigh fading [14]. Moreover, when the phases of $V_{n}^{i j}$ are randomly selected, the covariance matrix approaches the identity matrix (see (18)), so the channel will be uncorrelated.

An arbitrarily correlated MIMO Rayleigh channel, with a given covariance matrix $\mathbf{R}$, can be easily modeled from the corresponding uncorrelated channel. Given the multi-path matrices $\mathbf{W}_{n}$ of the corresponding uncorrelated channel $\mathbf{H}_{w}$, the matrices of the correlated channel will be given by

$$
\operatorname{vec}\left(\mathbf{V}_{n}\right)=\mathbf{R}^{1 / 2} \operatorname{vec}\left(\mathbf{W}_{n}\right) .
$$

It is easy to check that the resulting channel has the desired correlation characteristics

$$
\begin{aligned}
\operatorname{vec}(\mathbf{H}) & =\sum_{n=1}^{N} \operatorname{vec}\left(\mathbf{V}_{n}\right) \exp \left(j \phi_{n}\right) \\
& =\sum_{n=1}^{N} \mathbf{R}^{1 / 2} \operatorname{vec}\left(\mathbf{W}_{n}\right) \exp \left(j \phi_{n}\right) \\
& =\mathbf{R}^{1 / 2} \operatorname{vec}\left(\mathbf{H}_{w}\right) .
\end{aligned}
$$

\section{B. Physical model of Ricean fading channels}

If the amplitude of one component, e.g. $V_{1}^{i j}$, is dominant (usually associated with the LOS wave) and the others have similar amplitudes (associated with the NLOS multi-path waves), the distribution of $\left|h^{i j}\right|$ will be Ricean [14]. The corresponding Ricean-K factor will be given by

$$
K^{i j}=\frac{E\left[\left|h_{L O S}^{i j}\right|^{2}\right]}{E\left[\left|h_{N L O S}^{i j}\right|^{2}\right]}=\frac{\left|V_{1}^{i j}\right|^{2}}{\sum_{n=2}^{N}\left|V_{n}^{i j}\right|^{2}} .
$$

If the NLOS components are correlated according to (24), we have the corresponding MIMO correlated Ricean channel. 


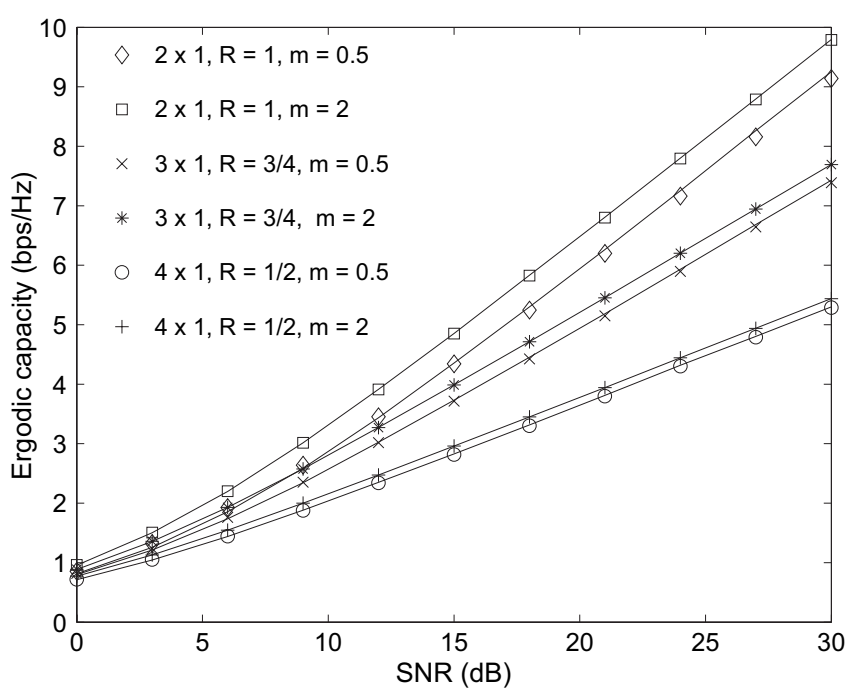

Fig. 1. Ergodic capacity of uncorrelated Nakagami-m channels as a function of the average SNR at the receiver (before decoding). It compares the predictions of (10) (solid curves) with Monte Carlo simulations (markers). The figure shows curves for different number of transmit antennas, code rates $(R)$ and Nakagami-m parameters.

\section{Vi. Simulation Results}

Now we compare the results of (10) with Monte Carlo simulations in different fading channels. We present results from both analytical and physical channel models. In the first case the variance of $\|\mathbf{H}\|_{F}^{2}$ was calculated using (11) or (13). For physical channel models the variance was calculated from (23). The figures show the ergodic capacity versus the SNR at the receiver (before decoding) for different number of antennas, code rates and fading distributions. In all cases, the solid lines represent the predictions of (10) and the markers are the values obtained by Monte Carlo simulations.

Figure 1 shows the ergodic capacity for different uncorrelated balanced Nakagami MIMO channels with one receive antenna and different numbers of transmit antennas and code rates. Two values of the Nakagami parameter have been considered: $m=0.5,2$. The variance of $\|\mathbf{H}\|_{F}^{2}$ was calculated using (13) and (14). In all cases the approximation (solid curves) is a good fit to the Monte Carlo values (markers). The maximum approximation error was $0.12 \mathrm{~dB}$ for the $2 \times 1$ configuration with $m=0.5$.

Figure 2 presents results for uncorrelated balanced MIMO Rayleigh channels with different number of transmit and receive antennas, and different code rates. The variance of $\|\mathbf{H}\|_{F}^{2}$ was also calculated using (13) and (14) with the $m_{i j}=1$. Once again the approximation closely matches the ergodic capacity with an approximation error lower than 0.015 $\mathrm{dB}$ in all cases.

The proposed approximation can also be used when the channel matrix entries have different fading distributions. For example Figure 3 compares the analytical prediction of (10) with Monte Carlo simulations for various uncorrelated Weibull fading channels where the channel matrix entries fade with different Weibull parameters. The variance of $\|\mathbf{H}\|_{F}^{2}$ was calculated using (13) and (16). In the configurations with two transmit antennas the Weibull parameters were $\beta_{i 1}=$ $1.5, \beta_{i 2}=5.0, i=1, \ldots n_{R}$. In the configurations with four

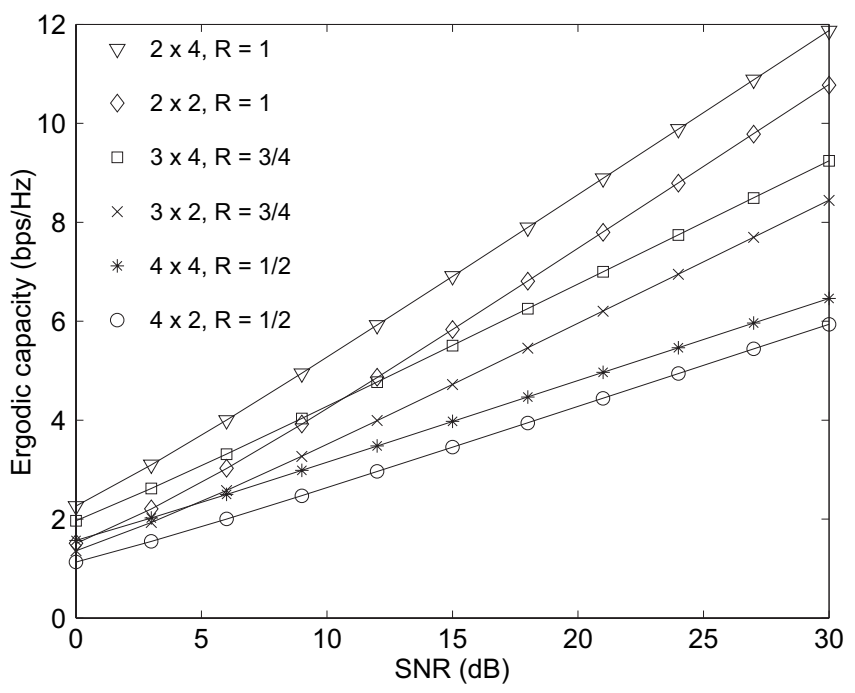

Fig. 2. Comparison between the analytical predictions of (10) (solid lines) and Monte Carlo simulations (markers) for uncorrelated MIMO Rayleigh channels with different number of antennas and code rates.

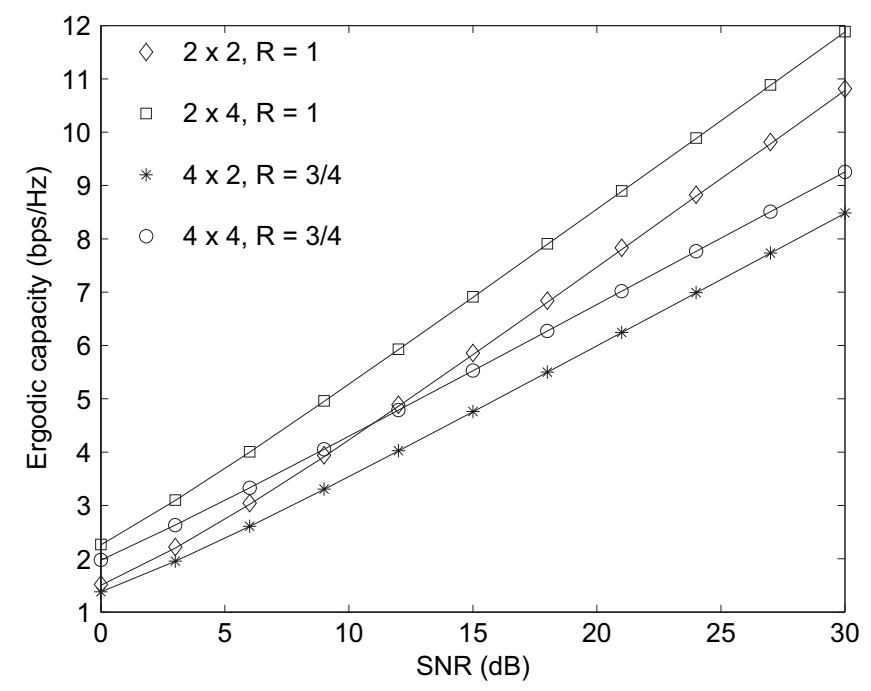

Fig. 3. Comparison between the analytical predictions of (10) (solid lines) and Monte Carlo simulations (markers) for uncorrelated MIMO Weibull channels where the channel matrix entries have different Weibull fading parameters.

transmit antennas the fading parameters were $\beta_{i 1}=1.5, \beta_{i 2}=$ $2.0, \beta_{i 3}=4.0, \beta_{i 4}=5.0, i=1, \ldots n_{R}$. The mean power gains $(\Omega i j)$ were identical in all cases. The maximum approximation error was always lower than $0.04 \mathrm{~dB}$.

To analyze the accuracy of (10) using realistic covariance matrices we consider the spatial correlation model of [15]. This is a 'one-ring' model suitable for NLOS propagation scenarios where the transmitter is not obstructed by local scatterers and the receiver is surrounded by a ring of local scatterers of radius $L$. Assuming linear arrays perpendicular to the line between the transmitter and the receiver, the entries of the covariance matrix are given by

$$
\rho^{i j, k s}=J_{0}\left(2 \pi d_{i k} / \lambda\right) J_{0}\left(2 \pi \arcsin (L / D) d_{j s} / \lambda\right)
$$

where $J_{0}(x)$ is the zeroth-order Bessel function of the first kind, $\lambda$ is the wavelength, $D$ is the distance between the transmitter and the receiver and $d_{i k}$ and $d_{j s}$ are the distances 


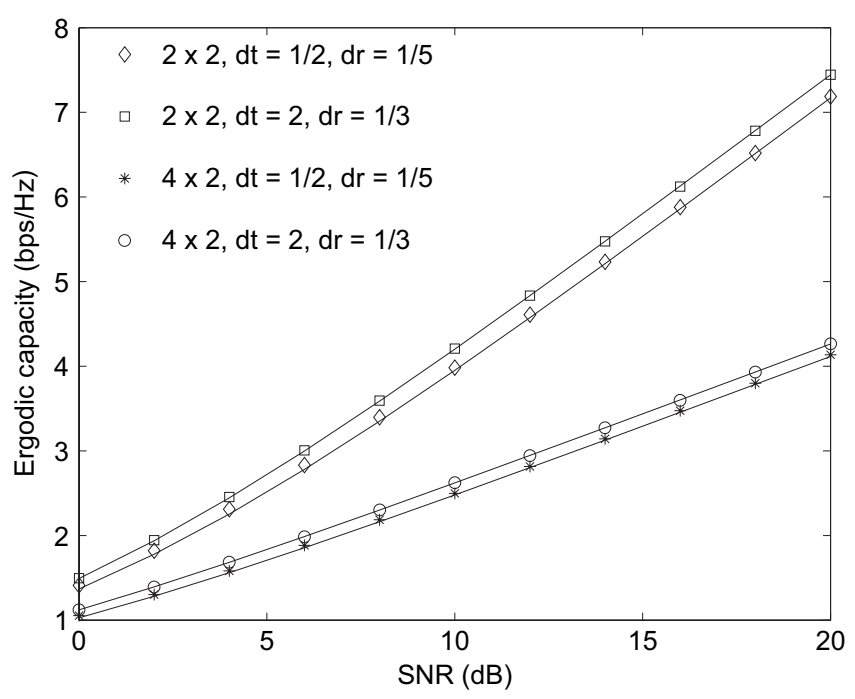

Fig. 4. Ergodic capacity of different correlated MIMO Rayleigh channels. $d t$ and $d r$ are the distance between adjacent antennas in wavelengths in the transmit and receive linear arrays, respectively. The graph compares the analytical predictions of (10) (solid lines) with Monte Carlo simulations (markers).

between the antennas in the receive and transmit arrays, respectively. In the simulations we have considered $L / D=$ 0.15 which is a typical value in microcellular environments.

Figure 4 shows the ergodic capacity for correlated balanced MIMO Rayleigh channels modeled as multi-path channels (see (24)). The covariance matrices were calculated using (27). In all cases, the multi-path channels were modeled with $N=100$ waves. The variance of $\|\mathbf{H}\|_{F}^{2}$ was calculated from (23). The figure shows curves for MIMO configurations with different number of antennas and with different antenna spacings in the linear arrays. The code rates were 1 and $1 / 2$ in the configurations with two and four transmit antennas, respectively. $d_{t}$ and $d_{r}$ denote the distance in wavelengths between adjacent antennas in the transmit and receive arrays, respectively. The figure shows that (10) closely matches the ergodic capacity regardless of the SNR, number of antennas and correlation conditions. The maximum approximation error was of $0.06 \mathrm{~dB}$ for the $2 \times 2$ configuration with lower antennas separation.

Identical results were obtained when the variance of $\|\mathbf{H}\|_{F}^{2}$ was calculated from (11) using directly the power covariance matrices of the MIMO Rayleigh channels. In this case the covariance matrices and power covariance matrices are related by $\mathbf{R}=\sqrt{\mathbf{R}_{P}}$, where $\sqrt{\cdot}$ denotes the elementwise square root [12].

In Figure 5 we present results for correlated MIMO Ricean channels modelled as multi-path channels. In all cases the Ricean-K factor was $K=3$ and the distances between adjacent antennas in the transmit and receive linear arrays were $d t=\lambda$ and $d r=\lambda / 4$, respectively. In all cases the channels were modeled with $N=100$ waves. Once again, the approximation (10) is a good fit to the Monte Carlo simulations. The maximum approximation error was less than $0.04 \mathrm{~dB}$ in all cases.

The expressions (10) and (23) can also be used in conjunction with site-specific physical channel models. For example,

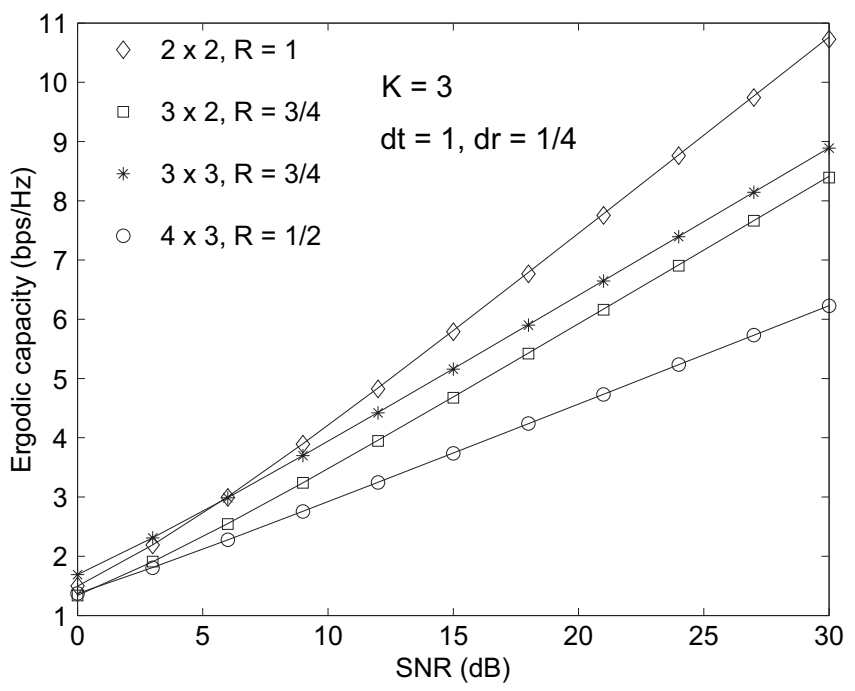

Fig. 5. Comparison between the predictions of (10) (solid lines) and Monte Carlo simulations (markers) for correlated MIMO Ricean channels with different number of antennas. In all cases the Ricean-K factor is $K=3$. The distances between adjacent antennas in the transmit and receive linear arrays are $d t=\lambda$ and $d r=\lambda / 4$, respectively.

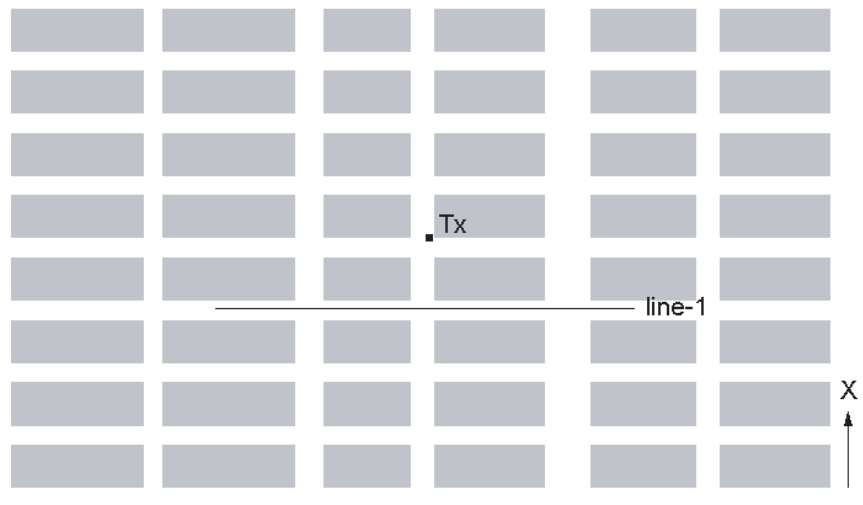

Fig. 6. Top view of the urban microcell showing the transmit array location. Line-1 shows the receive array locations in the simulations.

here we use a 3D ray-tracing propagation tool [16] to obtain the multi-path matrices in a specific urban microcellular scenario depicted in Figure 6. It consists of 48 regularly distributed buildings with uniform height $(25 \mathrm{~m})$ and rectangular sections forming a rectilinear grid of streets. This scenario represents an area of midtown Manhattan which has been traditionally used in the validation of a number of outdoor propagation models. The area of the environment is $900 \times$ $500 \mathrm{~m}^{2}$. In the simulations we assumed horizontal linear arrays of identical omnidirectional single-polarized antennas oriented along the $\mathrm{X}$ axis. The transmit and receive arrays had heights of $20 \mathrm{~m}$ and $1.5 \mathrm{~m}$, respectively. The simulations were carried out at $1.8 \mathrm{GHz}$ for different locations of the receiver array along line-1. The separations between the transmit and receive antennas were $6 \lambda$ and $\lambda$, respectively. To focus on the case where only small-scale fading is present, we assume perfect power control, so we set $\alpha=1$ in all the receiver locations. The average SNR was assumed to be $15 d B$ in all cases. Line1 comprises very different propagation conditions, including line-of-sight and non-line-of-sight locations. Also, the transmit 


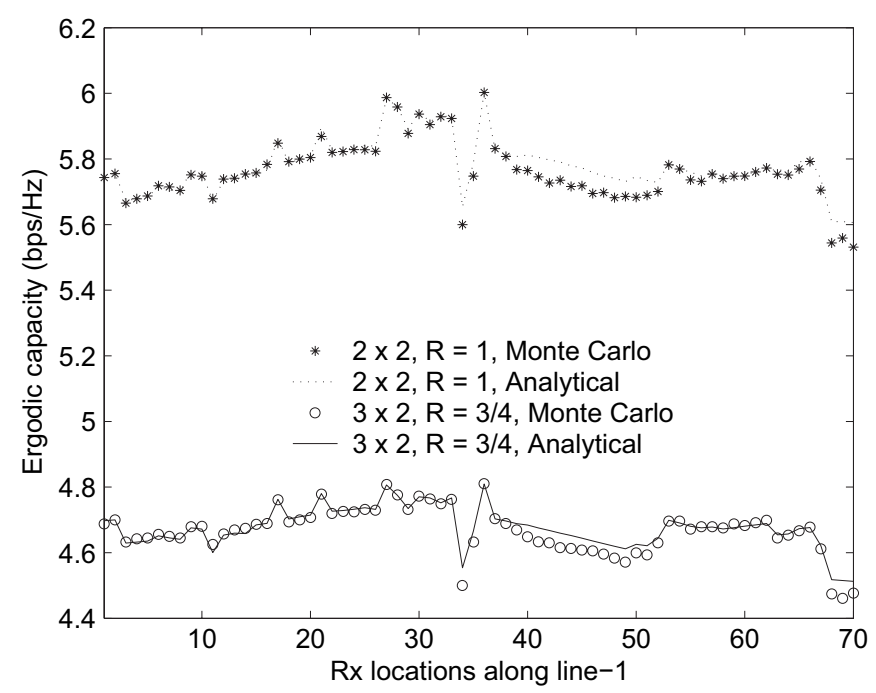

Fig. 7. Ergodic capacity for different receiver array locations along line-1 (see figure 6). The graph compares the predictions of (10) with Monte Carlo simulations for $S N R=15 d B$, two receive antennas and different number of transmit antennas.

and receive angular power spreads are very different along the points of line-1, producing a wide range of channel correlation conditions. Figure 7 compares the second-order analytical prediction by (10) with Monte Carlo simulations for $2 \times 2$ and $3 \times 2$ configurations with code rates $R=1$ and $R=3 / 4$, respectively. The maximum approximation error is $0.07 \mathrm{~dB}$. These results show that (10) constitutes a tight approximation to the ergodic capacity in a variety of propagation conditions.

\section{CONCLUSIONS}

In this letter we have derived a closed-form approximation to the ergodic capacity of narrow-band MIMO-OSTBC systems that is valid for any fading channel. The expression is a simple closed-form function of the variance of the squared Frobenius norm of the channel matrix, which equals the sum of the entries of the power covariance matrix. Therefore, the power covariance matrix is the only channel statistic needed for the capacity estimation. In the case of uncorrelated channels only the power variances of the channel matrix entries are required. These channel statistics can be easily obtained from both analytical and physical MIMO channel models, so the approximation can be used in conjunction with both types of channel modeling. The proposed expression is quite tight regardless of the fading distributions, correlation conditions, number of antennas and SNR regime. The accuracy of the approximation suggests that only the knowledge of the power covariance matrix is necessary for a tight estimation of the ergodic capacity. In the case of uncorrelated channels, it implies that the variance of the corresponding power distribution is enough for a tight estimation of the ergodic capacity. Unlike other proposed expressions, this approximation can be used for MIMO channels when the channel matrix entries have different fading distributions. This situation typically arises in distributed communication networks employing cooperative STBC transmission.

\section{ACKNOWLEDGMENT}

This work has been partially supported by Spanish Ministry of Education and Science under project TEC2004-06451-C0502 .

\section{REFERENCES}

[1] S. Alamouti, "A simple transmit diversity technique for wireless communications," IEEE J. Sel. Areas Commun., vol. 16, no. 8, pp. 14511458 , Oct. 1998.

[2] V. Tarokh, H. Jafarkhani, and A. R. Calderbank, "Space-time block codes from orthogonal designs," IEEE Trans. Inf. Theory, vol. 45, no. 5, pp. 14561467, July 1999.

[3] S. Sandhu, R. Heath, and A. Paulraj, "Space-time block codes versus space-time trellis codes," in Proc. IEEE ICC 2001, vol. 4, pp. 11321136.

[4] R. U. Nabar, H. Bolcskei, and A. J. Paulraj, "Outage properties of spacetime block codes in correlated Rayleigh or Rician fading environments," in Proc. IEEE ICASSP 2002, pp. 23812384.

[5] L. Musavian, M. Dohler, M. R. Nakhai, and A. H. Aghvami, "Closedform capacity expressions of orthogonalized correlated MIMO channels," IEEE Commun. Lett., vol. 8, no. 6, pp. 365367, June 2004.

[6] M. Dohler and H. Aghvami, "Information outage probability of distributed STBCs over Nakagami fading channels," IEEE Commun. Lett., vol. 8, pp. 437439, July 2004.

[7] E. G. Larsson and P. Stoica, Space-Time Block Coding for Wireless Communications. Cambridge University Press, 2003.

[8] H. Zhang and T. A. Gulliver, "Capacity and error probability analysis for orthogonal space-time block codes over fading channels," IEEE Trans. Wireless Commun., vol. 4, pp. 808819, March 2005.

[9] H. C. Espinosa, J. R. Fonollosa, and J. A. D. Penin, "Channel capacity of space-time block coding," in Proc. IST Mobile Communications Summit 2001, pp. 914.

[10] S. Sandhu and A. Paulraj, "Space-time block codes: a capacity perspective," IEEE Commun. Lett., vol. 4, no. 12, pp. 384386, Dec. 2000.

[11] T. M. Apostol, Mathematical Analysis. Addison Wesley Publishing Company, 1974.

[12] J. W. Wallace and M. A. Jensen, "Modeling the indoor MIMO wireless channel," IEEE Trans. Antennas Propag., vol. 50, pp. 591599, May 2002.

[13] X. Li, M. Chen, and W. Liu, "Application of STBC-encoded cooperative transmissions in wireless sensor networks," IEEE Signal Proccesing Lett., vol. 12, no. 2, pp. 134137, Feb. 2005.

[14] G. D. Durgin, Space-Time Wireless Channels. Upper Saddle River, NJ: Prentice Hall PTR, 2002.

[15] D. Shiu, G. J. Foschini, M. J. Gans, and J. M. Kahn, "Fading correlation and its effect on the capacity of multielement antenna systems," IEEE Trans. Commun., vol. 48, pp. 502513, March 2000.

[16] M. F. Catedra and J. Perez, Cell Planning for Wireless Communications. Norwood, MA: Artech House, 1999. 